IRA-International Journal of Applied Sciences

ISSN 2455-4499; Vol.04, Issue 01 (2016)

Institute of Research Advances

http://research-advances.org/index.php/IRAJAS

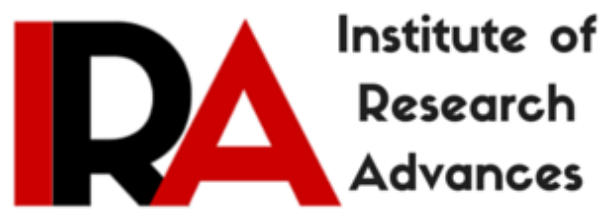

\title{
Butterfly diversity of Satara Tehsil, District Satara Maharashtra
}

\author{
Pawar P. A. ${ }^{1}$, Deshpande V Y ${ }^{2}$ \\ 1. Research Scholar, Shri Jagdish Prasad Jhabarmal Tibrewala \\ University,Jhunjhunu, Rajasthan-333001, India. \\ 2. Department of Zoology, Yashwantrao Chavan Institute of Science Satara, \\ Maharashtra 45001, India.
}

DOI: http://dx.doi.org/10.21013/jas.v4.n1.p16

\section{How to cite this paper:}

P. A., P., \& V Y, D. (2016). Butterfly diversity of Satara Tehsil, District Satara

Maharashtra. IRA-International Journal of Applied Sciences (ISSN 2455-4499), 4(1).

doi:http://dx.doi.org/10.21013/jas.v4.n1.p16

(C) Institute of Research Advances

(c)) EY-NO

This works is licensed under a Creative Commons Attribution-Non Commercial 4.0 International License subject to proper citation to the publication source of the work.

Disclaimer: The scholarly papers as reviewed and published by the Institute of Research Advances (IRA) are the views and opinions of their respective authors and are not the views or opinions of the IRA. The IRA disclaims of any harm or loss caused due to the published content to any party. 


\section{ABSTRACT}

Butterflies and moths belong to the order Lepidoptera (scaly winged insects) The butterfly species of family Nymphalidae were identified as indicators of disturbance in any area. In the present study a total of 52 individuals belonging to 9 sub families 28 genera were identified and were recorded. Among which Limenitidiae was found to be the most dominant subfamily followed bySatyrinae. The area of study having rich diversity of butterflies, therefore this study may be of great importance for the conservation ofbutterflies. This study being the pioneer study in the area is of immense importance.

Keywords: Satara Tehsil, butterfly diversity, Abundance, Biodiversity,conservation.

\section{Introduction}

Lepidoptera is the second largest order in the class Insecta. Lepidoptera are regarded as one of the important component of biodiversity (New and Collins, 1991) and are the second largest order among insects made up of approximately 1, 50,000 species so far known to the literature. These include moth (Hetrocera) and butterflies (Rhopalocera) of which 70,820 are butterflies according to more recent estimate (Shields, 1989) . Although several estimate have been made from time to time, ranging from a low of 13,000 (Owen, 1971) to the maximum of 20,000 (Vane Wrights, 1978)

The butterflies are very well known for their beauty as they bear beautiful wings of various colours .The degree of diversity depends upon the adaptability of aspecies to a particular micro habitat. The Indian subcontinent (CISC) has about 1439 species of butterflies out of which 100 species are endemic to it and at least 26 taxa are today globally threatened as per the IUCN (1990) Red List of threaten animals and insects (Singh and Pandey, 2004). According to Gaonkar (1996) India hosts 1,501 species of butterflies, of which peninsular India hosts 350, and the Western Ghats, 331. Recent data indicates 1318 species in India according to Varshney and Smetacek (2015)

Butterflies are sensitive biota which gets severely affected by the environmental variation and changes in the forest structure as they are closely dependent on plants (Pollard 1990 and Blair, 1999) . They also react quickly to any kind of disturbance and changes in the habitat quality making a good indicator to study changes in the habitat and landscape structure variations (Blair,1999) In this study an attempt is made to understand how the distribution and variation in butterfly diversity changes with habitats in various sites in the Northern Western Ghats. An area rich in biodiversity is of great importance for conservation.

Butterflies are highly reactive and respond to slightest changes in their habitat. Hence the conservation of habitats and butterflies must be given priority (Sidhu;2011) .

The forests are lost at higher rate in Asia (Sodhi et al., 2010) due tologging (Lambert \& Collar, 2002) agricultural (Koh\&Wilcove, 2008) even endemic regions (Sodhi et al., 2010) . Effects in diversity if vertebrates (e.g., Chiarello, 1999; Stouffer et al., 2006) to smaller ratio on insects (e.g.,Didham et al., 1996) .

In Maharashtra various workers like Kunte (1997) , (2001) .Rane\&Ranade (2004) ,Padhye et al. (2006) , (2009) Tiple et al. (2009), (Margules\&Pressey 2000) ,.

Nymphalidae contains around 6000 species ( Nieukerken et al.,2011), largest family of true butterflies. The family originated around 94 MYA in the mid Cretaceous. Diversifica- tion of the group began in the Late Cretaceous (current subfamilies) appeared shortly after the CretaceousPaleogene (K-Pg) boundary(Heikkilä et al. 2012) .One of the best studied family.27\% ofNymphalid butterfly species in Himalaya are endemic (Evans, 1932; Saikia, 2008) .

\section{Study Area}

The present study was carried out at Satara Thesil during the calendar year 2013 to 2016. Satara Thesil lies between (170.5' to $18^{0} .1^{\prime} \mathrm{N}$ latitude and $073^{\circ} 33^{\prime} 74.76^{\prime} \mathrm{E}$ longitude) is located on the Western Ghats of India in the State of Maharashtra. Satara is located about $110 \mathrm{~km}$ away from Pune City in a south-westerly direction. The family Nymphalidae was studied so as to study the diversity in detail. The study area was regularly searched during the morning and evening hrs for the activity of (Image 1) . 
The flora of Satara is endemic as it is a part of the Northern Western Ghats. The average annual rainfall for the year 2014-2016 as provided by the Regional Meteorological Centre, Mahableshwar .

The Arabian Sea brings in the mansoon rain early March and lasts till June. April and May are the hottest months, the average maximum temperature being $40^{\circ} \mathrm{C}\left(104^{\circ} \mathrm{F}\right)$. Monsoon begins in late June and the city receives about 800 millimeters (31 in) of rain by the end of September, with the average maximum being $32^{\circ} \mathrm{C}\left(90^{\circ} \mathrm{F}\right)$ during those months. October and November see the retreat of the monsoon and a return of high temperatures till late November. Winter starts in December and ends in late February with average temperatures of around $23^{\circ} \mathrm{C}\left(73^{\circ} \mathrm{F}\right)$. The climatic seasons viz. premonsoon, monsoon, post-monsoon and winter could be considered. The variations in seasons have been seen in last few years.

\section{Material and Method}

Biodiversity of butterfly family Nymphalidae had beensurveyed opportunistically Butterflies were identified directly in the field visually with the help of field guides. Collection was restricted to those specimens that could not be identified directly. All the scientific names follow Varshney, R K \&Smetacek P (2015) A Synoptic Catalogue of the Butterflies of India and common English names follow Wynter-Blyth .Benthum\&Hooker (1862) system of classification is followed for plants. The study area was fully explored during the period from March 2014 to January 2016 (Image no. 1).The study area was visited twice in each season during above period.

Pre monsoon: March, April; Monsoon: June, July, August and September;Post-monsoon:October and November; Winter: December, January and February. 


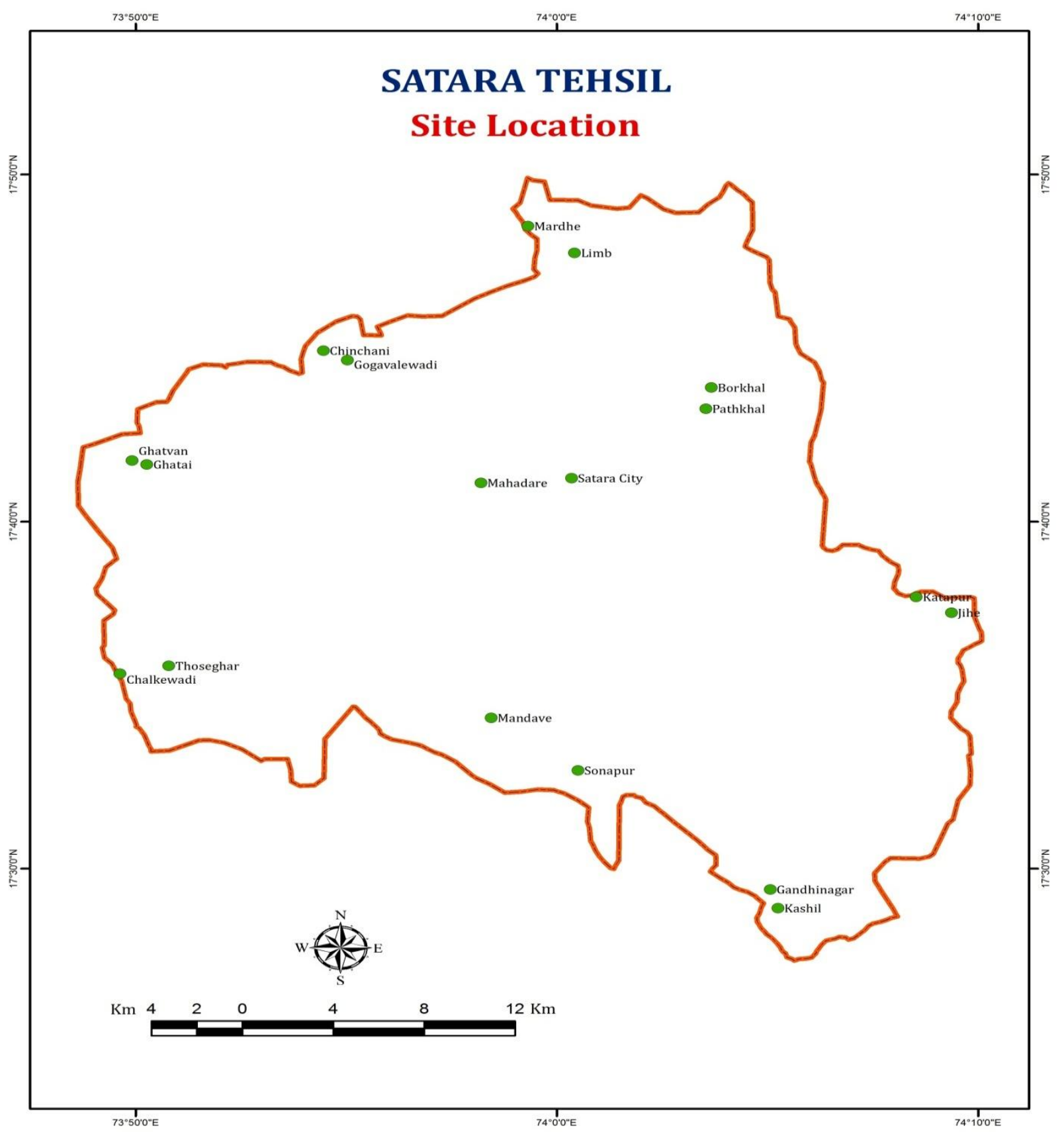

Image: 1 showing the sites where butterfly diversity was studied in Satara Tehsil.

Table no. 1. Site locations.

\begin{tabular}{|l|l|l|}
\hline Sr. No & Site Location & Assigned Name \\
\hline 01 & Chinchani-Gogavalewadi & A \\
\hline 02 & Mahadare-Satara City & B \\
\hline 03 & Chalkewadi-Thoseghar & C \\
\hline 04 & Mandave-Sonapur & E \\
\hline 05 & Ghatvan-Ghatai & F \\
\hline 06 & Jihe-Katapur & G \\
\hline 07 & Pathkal-Borkhal & H \\
\hline 08 & Mardhe-Limb & I \\
\hline 09 & Kashil-Gandhinagar & \\
\hline
\end{tabular}




\section{Results and Observation}

The family Nymphalidae is well distributed in the Satara tehsilwith 52species. Three species are endemic to the Western Ghats, 6 species have shared endemism of Peninsular India and 2 are endangered.

During the course of study, 52 species of butterflies belonging to the family Nymphalidae were recorded in Satara Tehsil as recorded in (table no 3) In the present study a total of 52 individuals belonging to 11 sub families were identified and 28 genera were recorded.(table no 3) Among which Limenitidiae was found to be the most dominant sub family followed by Satyrinae. The area of study having rich diversity of host plants being a part of Western Ghats following are the species recorded.

The Sub family Danainae has five species belonging to two tribes and two genera. The Tribe danainihas Genus Danaus (Kluk,1780) represented by Danaus.chrysippus.chrysippus Plain tiger, (Danaus.genutia. genutia) Common tiger, (Parantica .aglea.aglea) Glassy tiger, (Tirumala.liminace.exociticus) Blue tiger .TheTribeEuploeini Genus Euploea (Euploea. core.core) Common Crow.

The sub family Charaxinae is represented by one tribe Charaxin and two genera.The Genus Polyura(Polyura.agraria.agraria) Anomalous Nawab (Polyura. athamas.athamas.) Common NawabThe Genus Charaxes is represented by (Charaxes .psaphon.imna) Plain Tawny Rajah and (Charaxes .solon.solon) Black Rajah.

The sub family Satyrinae is the second largest sub family represented by two tribes and four genera.The tribe Tribeni and Melanitini are represented by four genera Elymnias, Melanitini, Lethe,Ypthima Genus Elymnias (Elymniashypermnestra.caudata) Common Palmfly Tribe Melanitini Genus Melanitis (Melanitis.leda.ledaenus) Common Evening Brown (Melanitis.phedima.varaha) Dark Evening Brown,Genus Lethe (Lethe.europa.ragalav) Bamboo Tree Brown, (Lethe.rohria. neelgheriensis) Common Treebrown(Mycalesis .perseus )Common Bushbrown Genus Ypthima (Ypthima .asterope. maahratta) Common Threering (Ypthima .baldus.madrasa) Common Fivering(Ypthima .huebneri. huebneri) Common Fourring(Ypthima .philomela.tabella) Baby Fivering.

The sub family Limenitidina is the largest sub family has a single Tribe limenitidinihaving seven genera Genus Neptis(Neptis.hylas.varmona) CommomnSailer, (Neptis.jumbha .jumbha) Chestnut-Streaked Sailer.GenusPantoporia (Pantoporia.hordonia.hordonia) Common Lascar(Pantoporia. sandaca. davidsoni.) Extra Lascar Genus Athyma (Athyma.nefte.inara) Colour Sergeant, (Athyma.perius.perius.) Common Sergeant.GenusModuza.(Moduza .procris.undifragus.) Commander, Genus Cynitia (Cynitia.lepideamiyana.) Grey Count .Genus Euthalia (Euthaliaaconthea.anagama.) Common Baron (Euthaliaaconthea,meridionalis) CommoBaron (Euthalialubentina.arasada) Gaudy Baron (Euthalia. lubentina. lubentina) Gaudy BaronGenus (SymphaedraSymphaedranasi) Baronate.

The sub family Heliconiinae is represented by Tribe HrliconiiniGenus Phalanta(Phalanta.phalanta.phalanta) Common Leopard,GenusCupha(Cupha .erymanthis.maja.) Rustic.

The Sub family Biblidinae consist of the Tribe BiblidiniGenus Ariadne(Ariadne. ariadne. indica) Angled Castor, (Ariadne.merione.merione) Common Castor,GenusByblia(Byblia.ilithyia) Joker.

Sub family Apaturinae Tribe Apaturini Genus Rohana(Rohana.parisatis .atacinus) Black Prince, Genus Euripus ( Euripus.consimilis.meridionalis) Painted Courteasan .

Sub family Cyrestinae Tribe Cyrestini Genus Cyrestis (Cyrestis.thyodamanas.indica)Common Map.

Sub family Nymphalinae Tribe NymphaliniGenus Venessa(Venessa .cardui.cardui.) Painted Lady,TribeJunoniini Genus Junonia (Junonia.almana almanac) Peacock Pansy, (Junonia iphita. pulviatalis) Chocolate Pansy,(Junonia .lemonias.vaisya) Lemon Pansy, (Junonia orithya.swinhoei.) 
Blue Pansy(Junonia.hierta.hierta) Yellow Pansy(Junonia .atlites,atlites) Grey Pansy Tribe Kallimini Genus Hypolimnas(Hypolimnas.bolina,jacintha) Great Eggfly,(Hypolimnas.misippus.) DanaidEggfly, Genus Kallima(Kallimahorsfieldi) Southern Blue Oakleaf,The sub family Acraeinae represents Tribe AcraeiniGenusAcraea (Acraea.violae) Tawny Coster.The sub family Libytheinae Genus Libythea (Libythea.myrrha.rama) Club Beak.(table no 3)

Table no 2. Site wise distribution of species (site names as per table no 1).

\begin{tabular}{|c|c|c|c|c|c|c|c|c|c|c|c|}
\hline \begin{tabular}{l|l} 
Sr \\
no
\end{tabular} & Scientific name & $\begin{array}{l}\text { Common } \\
\text { Name }\end{array}$ & $\mathbf{A}$ & B & $\mathbf{C}$ & D & $\mathbf{E}$ & $\mathbf{F}$ & $\mathbf{G}$ & $\mathbf{H}$ & $\mathbf{I}$ \\
\hline 1) & $\begin{array}{l}\text { Genus (kluk,1780) } \\
\text { Danaus.chrysippus }\end{array}$ & Plain tiger & + & + & + & + & + & + & + & + & + \\
\hline 2) & Danaus.genutia & Common tiger & + & + & + & + & + & + & + & + & + \\
\hline 3) & Parantica .aglea & Glassy tiger & + & + & + & + & + & + & + & + & + \\
\hline 4) & Tirumala.liminace & Blue tiger & + & + & + & + & + & + & + & + & + \\
\hline 5) & \begin{tabular}{|l} 
Genus Idea \\
Idea.malabarica.kanarensis
\end{tabular} & $\begin{array}{l}\text { Malbar Tree } \\
\text { Nymph }\end{array}$ & & & + & & + & & & & \\
\hline 6) & \begin{tabular}{|l|} 
Genus Euploea \\
Euploea .core.core \\
\end{tabular} & Common Crow & + & + & + & + & + & + & + & + & + \\
\hline 7) & Euploea .sylvester.coreta & $\begin{array}{l}\text { Double-branded } \\
\text { crow }\end{array}$ & & & + & & + & & & & \\
\hline 8) & $\begin{array}{l}\text { Genus Polyura } \\
\text { Polyura.agraria.agraria }\end{array}$ & $\begin{array}{l}\text { Anomalous } \\
\text { Nawab }\end{array}$ & & & + & & + & & & & \\
\hline 9) & Polyura.athamas.athamas.. & $\begin{array}{l}\text { Common } \\
\text { Nawab }\end{array}$ & + & + & + & & + & & & & \\
\hline 10 & $\begin{array}{l}\text { Genus Charaxes } \\
\text { Charaxes .psaphon.imna }\end{array}$ & $\begin{array}{l}\text { Plain Tawny } \\
\text { Rajah }\end{array}$ & & & + & & + & & & & \\
\hline 11 & Charaxes solon.solon & Black Rajah & + & + & + & & + & & & & \\
\hline 12 & $\begin{array}{l}\text { Genus Elymnias } \\
\text { Elymniashypermnestra.caudata }\end{array}$ & $\begin{array}{l}\text { Common } \\
\text { Palmfly }\end{array}$ & & + & & & + & & & & \\
\hline 13 & \begin{tabular}{|l|} 
Genus Melanitis \\
Melanitis.leda.ledaenus
\end{tabular} & $\begin{array}{l}\text { Common } \\
\text { Evening Brown }\end{array}$ & + & + & + & + & + & + & + & + & + \\
\hline 14 & Melanitis.phedima.varaha & $\begin{array}{l}\text { Dark Evening } \\
\text { Brown }\end{array}$ & & & + & & + & & & & \\
\hline 15 & $\begin{array}{l}\text { Genus Lethe } \\
\text { Lethe .europa.ragalava }\end{array}$ & $\begin{array}{l}\text { Bamboo Tree } \\
\text { Brown }\end{array}$ & & & + & & + & & & & \\
\hline 10 & Lethe rohria.neelgheriensis. & $\begin{array}{l}\text { Common } \\
\text { Treebrown }\end{array}$ & + & + & + & + & + & + & + & + & + \\
\hline 11 & Mycalesis .perseus.tabitha & $\begin{array}{l}\text { Common } \\
\text { Bushbrown }\end{array}$ & + & + & + & + & + & + & + & + & + \\
\hline 18 & Mycalesis .mineus.polydecta & $\begin{array}{l}\text { Dark brand } \\
\text { Bushbrown }\end{array}$ & & & + & + & & + & & & \\
\hline 19 & $\begin{array}{l}\text { Genus Ypthima } \\
\text { Ypthima .asterope.maahratta }\end{array}$ & $\begin{array}{l}\text { Common } \\
\text { Threering }\end{array}$ & & & + & + & & + & & & \\
\hline 20 & Ypthima .baldus.madrasa & $\begin{array}{l}\text { Common } \\
\text { Fivering }\end{array}$ & + & + & + & + & + & + & + & + & + \\
\hline 21 & Ypthima.huebneri.huebneri & $\begin{array}{l}\text { Common } \\
\text { Fourring }\end{array}$ & + & + & + & + & + & + & + & + & + \\
\hline 22 & Ypthima .philomela.tabella & Baby Fivering & & + & + & & + & & & & \\
\hline 23 & $\begin{array}{l}\text { Genus Neptis } \\
\text { Neptis.hylas.varmona }\end{array}$ & C ommon Sailer & + & + & + & + & + & + & + & + & + \\
\hline 24 & Neptis.jumbha.jumbha & $\begin{array}{l}\text { Chestnut- } \\
\text { Streaked Sailer }\end{array}$ & & + & + & & + & & & & \\
\hline 25 & $\begin{array}{l}\text { Genus Pantoporia } \\
\text { Pantoporia,hordonia.hordonia }\end{array}$ & CommonLascar & & + & + & & + & & & & \\
\hline $2 \theta$ & Pantoporia.sandaca.davidsoni. & Extra Lascar & & + & + & & + & & & & \\
\hline
\end{tabular}


IRA-International Journal of Applied Sciences

\begin{tabular}{|c|c|c|c|c|c|c|c|c|c|c|c|}
\hline 27 & $\begin{array}{l}\text { Genus Athyma } \\
\text { Athyma.nefte.inara }\end{array}$ & Colour Sergeant & & & + & & + & & & & \\
\hline 28 & Athyma.perius.perius. & $\begin{array}{l}\text { Common } \\
\text { Sergeant }\end{array}$ & & + & + & + & + & & & & \\
\hline 29 & $\begin{array}{l}\text { Genus Moduza } \\
\text { Moduza .procris.undifragus. }\end{array}$ & $\begin{array}{l}\text { Comman- } \\
\text { der }\end{array}$ & & + & + & & + & & & & \\
\hline 30 & $\begin{array}{l}\text { Genus Cynitia } \\
\text { Cynitia.lepideamiyana. }\end{array}$ & Grey Count & & & + & & + & & & & \\
\hline 31 & $\begin{array}{l}\text { Genus Euthalia } \\
\text { Euthaliaaconthea.anagama. }\end{array}$ & Common Baron & + & + & + & + & + & + & + & + & + \\
\hline 32 & Euthalia.lubentina.lubentina & Gaudy Baron & & + & + & + & + & + & & & \\
\hline 33 & $\begin{array}{l}\text { Genus Symphaedra } \\
\text { Symphaedranasi. }\end{array}$ & Baronate & + & + & & & & & & & \\
\hline 34 & $\begin{array}{l}\text { Genus Phalanta } \\
\text { Phalanta.phalanta.phalanta }\end{array}$ & $\begin{array}{l}\text { Common } \\
\text { Leopard }\end{array}$ & + & + & + & + & + & & & & \\
\hline 33 & $\begin{array}{l}\text { Genus Cupha } \\
\text { Cupha .erymanthis.maja. }\end{array}$ & Rustic & & + & + & + & + & & & & \\
\hline 36 & $\begin{array}{l}\text { Genus Ariadne } \\
\text { Ariadne.ariadne.indica }\end{array}$ & Angled Castor & & + & + & & + & & & & \\
\hline 37 & Ariadne.merione.merione & Common Castor & + & + & + & + & + & + & + & + & + \\
\hline 38 & $\begin{array}{l}\text { Genus Byblia } \\
\text { Byblia.ilithyia }\end{array}$ & Joker & + & + & + & + & + & + & + & + & \\
\hline 39 & $\begin{array}{l}\text { Genus Rohana } \\
\text { Rohana.parisatis .atacinus }\end{array}$ & Black Prince & & + & + & & + & & & & \\
\hline 40 & $\begin{array}{l}\text { Genus Cyrestis } \\
\text { Cyrestis.thyodamanas .indica }\end{array}$ & Common Map & + & + & + & + & + & + & + & + & + \\
\hline 41 & $\begin{array}{l}\text { Genus Venessa } \\
\text { Venessa .cardui }\end{array}$ & Painted Lady & + & + & + & + & + & + & + & + & + \\
\hline 42 & $\begin{array}{l}\text { Genus Junonia } \\
\text { Junonia.almana almanac }\end{array}$ & Peacock Pansy & + & + & + & + & + & + & + & + & + \\
\hline 43 & Junonia .iphita.pulviatalis & $\begin{array}{l}\text { Chocolate } \\
\text { Pansy }\end{array}$ & + & + & + & + & + & + & + & + & + \\
\hline 44 & Junonia.lemonias.vaisya & Lemon Pansy & + & + & + & + & + & + & + & + & + \\
\hline 45 & Junoniaorithya.swinhoei. & Blue Pansy & + & + & + & + & + & + & + & + & + \\
\hline 46 & Junonia.hierta.hierta & Yellow Pansy & + & + & + & + & + & + & + & + & + \\
\hline 47 & Juznonia .atlites, atlites & Grey Pansy & + & + & + & + & + & + & + & + & + \\
\hline 48 & $\begin{array}{l}\text { Genus Hypolimnas } \\
\text { Hypolimnas.bolina,jacintha }\end{array}$ & Great Eggfly & + & + & + & + & + & + & + & + & + \\
\hline 49 & Hypolimnas.misippus & Danaid Eggfly & + & + & + & + & + & + & + & + & + \\
\hline 50 & $\begin{array}{l}\text { Genus Kallima } \\
\text { Kallimahorsfieldi }\end{array}$ & $\begin{array}{l}\text { Southern Blue } \\
\text { Oakleaf }\end{array}$ & & + & + & & + & & & & \\
\hline 51 & $\begin{array}{l}\text { Genus Acraea } \\
\text { Acraea.violae }\end{array}$ & Tawny Coster & & + & + & + & & & & & \\
\hline 52 & $\begin{array}{l}\text { Genus Libythea } \\
\text { Libythea .lepita.lepitoides }\end{array}$ & Club Beak & & + & + & + & & & & & \\
\hline
\end{tabular}

\section{Disscusion}

The Diversity pattern and faunal composition differ significantly between seasons

Species diversity was consistently higher during the monsoon season. There are some species found in close vicinity of the study area but not in the study area. The reason may be the humidity as the 
habitats and host plants are available in the study area yet the butterflies are absent. The highest diversity was recorded at the sites $\mathrm{B}$ and $\mathrm{C}$ (table no 1).The species are restricted to certain areas they have geographical and climatic needs and hence are of conservation value (Thomas 1991; VaneWright et al. 1991; Kakati 2006).

The microhabitat and the availability of the host plant are directly related to the butterfly diversity. The abundance and distribution is completely in the hands of climatic conditions like rainfall,temperature and humidity(Hill et al. 2003; Shubhalakshmi\&Chaturvedi, 1999) . This emphasizes the need for biodiversity assessments to cover sufficiently long period to account for seasonal variation in species abundance in different habitats. The species like the Common Sergeant (Athyma.perius.perius), Colour Sergeant. (Athyma. nefte.inara), Common Lascar (Pantoporia,hordonia.hordonia),Tawny Rajah (Charaxes.psaphon.imna) were recorded at high altitudinal sites and not observed in the low lying areas.(table no 2)

The species like Sailers,Leopards,Commanders,Extra laskars Chestnut Streaked Sailer(Neptis.jumbha.jumbha),CommonSailer (Neptis.hylas. varmona) CommonLeopard (Phalanta.phalanta.phalanta) Commander (Moduza.procris. undifragus) Extra Lascar (Pantoporia.sandaca. davidsoni) were common and abundant ( table no 2)

Our study reveals that there were butterflies which have been able to accept alternative host plants, butterflies which were capable of changing the host plant due to attitudinal variations .Based on the positive findings we have suggest that there is need for revision study.

Distributional status of the butterflies states that there were areas with high altitude where many sub families were abundant whereas there were areas where only one family was abundant.(table no 2). Some sites along the river bank sites indicated average abundance with no prominent sub family but equally scattered sub families. The status of a sub family cannot be stated in such a small scale.

A temporal and spatial study is required for the proper assessment of diversity for a long time scale. The seasonality of some species may be related toits host plant availability and dry weather condition. During winter season majority of the host plant become defoliated or no new foliages are appearing.

The seasonal patterns in tropical butterflies is unknown to correlate it with host plants (Wolda 1989) .The patterns of butterfly abundance may be a result of seasonal leaf sprouting, larval mortility, host plants in different microhabitats.(D' Amico \& Elkington 1995).

The study is concerned to butterfly diversity which are identified as indicators of ecosystem, slightest change in the habitat may lead to evolution or replacement of species (Wilson 1987; Liow et al. 2001) . Diversity assessments techniques are required to study the impact of habitat and seasonal variation. (Hamer et al. 2005) .

\section{Conclusion}

There is no work being done in Satara Thesil on butterfly diversity and if it is done it is not yet published hence this work is a pioneer work on butterflies of Satara Thesil. The purpose of generation of a authentic checklist of butterflies of family Nymphalidae has been done successfully .There are about 52 butterflies of the family Nymphalidae belonging to the Satara Thesil. The family Nymphalidae is a diverse one and most abundant among all other families.

\section{Acknowledgements:}

The authors are thankful to Mr Sunil H Bhoite \& MrMilind S Bhakare for their valuable knowledge and guidance, we are thankful to YCIS Satara \& Rayat Shikshan Sanstha for the financial support for this project. 
IRA-International Journal of Applied Sciences

Checklist

\begin{tabular}{|c|c|c|c|c|}
\hline Sr no & Sub Family & Tribe & Scientific name & Common name \\
\hline 1 & Dyainae- & danaini & $\begin{array}{c}\text { Genus (kluk,1780) } \\
\text { Danaus.chrysippus }\end{array}$ & Plain tiger \\
\hline 2 & & & Danaus.genutia & Common tiger \\
\hline 3 & & & Parantica .aglea & Glassy tiger \\
\hline 4 & & & Tirumala.liminace & Blue tiger \\
\hline 5 & & Euploeini & $\begin{array}{c}\text { Genus Idea } \\
\text { Idea.malabarica.kanarensis }\end{array}$ & Malbar Tree Nymph \\
\hline 6 & Charaxinae & Charaxin & $\begin{array}{c}\text { Genus Euploea } \\
\text { Euploea .core.core }\end{array}$ & Common Crow \\
\hline 7 & & & Euploea .sylvester.coreta & $\begin{array}{c}\text { Double-branded } \\
\text { crow }\end{array}$ \\
\hline 8 & & & $\begin{array}{c}\text { Genus Polyura } \\
\text { Polyura.agraria.agraria }\end{array}$ & Anomalous Nawab \\
\hline 9 & & & Polyura.athamas.athamas.. & Common Nawab \\
\hline 10 & Satyrinae & Tribeni & $\begin{array}{c}\text { Genus Charaxes } \\
\text { Charaxes .psaphon.imna }\end{array}$ & Plain Tawny Rajah \\
\hline 11 & & Melanitini & Charaxes .solon.solon & Black Rajah \\
\hline 12 & & & $\begin{array}{c}\text { Genus Elymnias } \\
\text { Elymniashypermnestra.caudata }\end{array}$ & Common Palmfly \\
\hline 13 & & & $\begin{array}{c}\text { Genus Melanitis } \\
\text { Melanitis.leda.ledaenus }\end{array}$ & $\begin{array}{l}\text { Common Evening } \\
\text { Brown }\end{array}$ \\
\hline 14 & & & Melanitis.phedima.varaha & Dark Evening Brown \\
\hline 15 & & & $\begin{array}{c}\text { Genus Lethe } \\
\text { Lethe .europa.ragalava }\end{array}$ & Bamboo Tree Brown \\
\hline 16 & & & Lethe .rohria.neelgheriensis. & Common Treebrown \\
\hline 17 & & & Mycalesis .perseus.tabitha & Common Bushbrown \\
\hline 18 & & & Mycalesis .mineus.polydecta & $\begin{array}{l}\text { Dark brand } \\
\text { Bushbrown }\end{array}$ \\
\hline 19 & & & $\begin{array}{c}\text { Genus Ypthima } \\
\text { Ypthima .asterope.maahratta }\end{array}$ & Common Threering \\
\hline 20 & Limenitidina & $\begin{array}{l}\text { Tribe } \\
\text { limenitidini }\end{array}$ & Ypthima .baldus.madrasa & Common Fivering \\
\hline 21 & & & Ypthima .huebneri.huebneri & CommonFourring \\
\hline 22 & & & Ypthima .philomela.tabella & Baby Fivering \\
\hline 23 & & & $\begin{array}{c}\text { Genus Neptis } \\
\text { Neptis.hylas.varmona }\end{array}$ & CommomnSailer \\
\hline 24 & & & Neptis.jumbha.jumbha & $\begin{array}{c}\text { Chestnut-Streaked } \\
\text { Sailer }\end{array}$ \\
\hline 25 & & & $\begin{array}{c}\text { Genus Pantoporia } \\
\text { Pantoporia,hordonia.hordonia }\end{array}$ & CommonLascar \\
\hline 26 & & & Pantoporia.sandaca.davidsoni. & Extra Lascar \\
\hline 27 & & & $\begin{array}{c}\text { Genus Athyma } \\
\text { Athyma.nefte.inara }\end{array}$ & Colour Sergeant \\
\hline 28 & & & Athyma.perius.perius. & Common Sergeant \\
\hline 29 & & & $\begin{array}{c}\text { Genus Moduza } \\
\text { Moduza .procris.undifragus. }\end{array}$ & Commander \\
\hline 30 & & & $\begin{array}{c}\text { Genus Cynitia } \\
\text { Cynitia.lepideamiyana. }\end{array}$ & Grey Count \\
\hline 31 & & & Genus Euthalia & Common Baron \\
\hline
\end{tabular}


IRA-International Journal of Applied Sciences

\begin{tabular}{|c|c|c|c|c|}
\hline & & & Euthaliaaconthea.anagama. & \\
\hline 32 & & & Euthalia.lubentina.lubentina & Gaudy Baron \\
\hline 33 & Heliconiinae & $\begin{array}{l}\text { Tribe } \\
\text { Hrliconiini }\end{array}$ & $\begin{array}{c}\text { Genus Symphaedra } \\
\text { Symphaedranasi. }\end{array}$ & Baronate \\
\hline 34 & & & $\begin{array}{c}\text { Genus Phalanta } \\
\text { Phalanta.phalanta.phalanta }\end{array}$ & Common Leopard \\
\hline 35 & Biblidinae & $\begin{array}{l}\text { Tribe } \\
\text { Biblidini }\end{array}$ & $\begin{array}{c}\text { Genus Cupha } \\
\text { Cupha .erymanthis.maja. }\end{array}$ & Rustic \\
\hline 36 & & & $\begin{array}{c}\text { Genus Ariadne } \\
\text { Ariadne.ariadne.indica }\end{array}$ & Angled Castor \\
\hline 37 & & & Ariadne.merione.merione & Common Castor \\
\hline 38 & Apaturinae & $\begin{array}{l}\text { Tribe } \\
\text { Apaturini }\end{array}$ & $\begin{array}{l}\text { Genus Byblia } \\
\text { Byblia.ilithyia }\end{array}$ & Joker \\
\hline 39 & & & $\begin{array}{c}\text { Genus Rohana } \\
\text { Rohana.parisatis .atacinus }\end{array}$ & Black Prince \\
\hline 40 & Cyrestinae & $\begin{array}{l}\text { Tribe } \\
\text { Cyrestini }\end{array}$ & $\begin{array}{c}\text { Genus Cyrestis } \\
\text { Cyrestis.thyodamanas .indica }\end{array}$ & Common Map \\
\hline 41 & Nymphalinae & $\begin{array}{l}\text { Tribe } \\
\text { Nymphalini }\end{array}$ & $\begin{array}{l}\text { Genus Venessa } \\
\text { Venessa .cardui }\end{array}$ & Painted Lady \\
\hline 42 & & $\begin{array}{l}\text { Tribe } \\
\text { Junoniini }\end{array}$ & $\begin{array}{c}\text { Genus Junonia } \\
\text { Junonia.almana almanac }\end{array}$ & Peacock Pansy \\
\hline 43 & & & Junonia .iphita.pulviatalis & Chocolate Pansy \\
\hline 44 & & & Junonia .lemonias.vaisya & Lemon Pansy \\
\hline 45 & & & Junoniaorithya.swinhoei. & Blue Pansy \\
\hline 46 & & & Junonia.hierta.hierta & Yellow Pansy \\
\hline 47 & & & Juznonia .atlites, atlites & Grey Pansy \\
\hline 48 & & $\begin{array}{l}\text { Tribe } \\
\text { Kallimini }\end{array}$ & $\begin{array}{c}\text { Genus Hypolimnas } \\
\text { Hypolimnas.bolina,jacintha }\end{array}$ & Great Eggfly \\
\hline 49 & & & Hypolimnas.misippus & DanaidEggfly \\
\hline 50 & & & $\begin{array}{c}\text { Genus Kallima } \\
\text { Kallimahorsfieldi }\end{array}$ & $\begin{array}{c}\text { Southern Blue } \\
\text { Oakleaf }\end{array}$ \\
\hline 51 & Acraeinae & $\begin{array}{l}\text { Tribe } \\
\text { Acraeini }\end{array}$ & $\begin{array}{l}\text { Genus Acraea } \\
\text { Acraea.violae }\end{array}$ & Tawny Coster \\
\hline 52 & Libytheinae & & $\begin{array}{c}\text { Genus Libythea } \\
\text { Libythea .lepita.lepitoides }\end{array}$ & Club Beak \\
\hline & & & & otal $=52$ \\
\hline
\end{tabular}

\section{References :}

1. Arora, G. S. (1990) . Collection and preservation of animals (Lepidoptera) .Zool.Surv. Ind., Calcutta, pp. 131-138.

2. Battist, A.( 1988) . Phytophagous insect in the energy flow of an artificial stand of PinusnigraArnold in northern Italy.Redia, 71(1) : 139-160.

3. Blair, Robert B. (1999) birds and butterflies along an urban gradient: surrogate taxa for assessing biodiversity?Ecological Applications 9: 164-170

4. Chandra, K., Chaudhary, L. K., Singh, R. K. and Koshta, M. L. (2002) .Butterflies of PenchTigerReserve, Madhya Pradesh. Zoo's Print Journal17(10) : 908-909.

5. Chandra, K., Sharma, R. M., Singh, A. and Singh,R. K. 2007. A checklist of butterflies of Madhya Pradesh and Chhattisgarh States,India. Zoos' Print Journal 22(8) : 2790-2798

6. Choudhury, K. 2010. Rediscovery of two rare butterflies Papilioelephenor Doubleday, 1845 and Shijimiamoorei Leech, 1889 from proposed Ripu-Chirang Wildlife Sanctuary, Assam, India. Journal of Threatened Taxa 2(4) : 831-834.

7. Gaonkar, H. (1996) . Butterflies of the Western Ghats with notes on those of Sri Lanka. A report to Centre forEcological Sciences, Indian Institute of Science, Bangalore; Zoological Museum, Copenhagen andNatural History Museum, London.

8. Ghosh, A. K. (1990) . Collection and Preservation of Animals. Zool. Surv. India, Calcutta, $71-80$. 
9. Hannyngton, F. (1910-1911) . The butterflies of Kumaon. Jour. Bombay Nat. Hist. Soc., 20 (3) : 871-872.

10. Jonathan, J. K. (1990) . Collection and preservation of animals (Hymenoptera) .Zoo.Surv.India, Calcutta, 147-150.

11. Joshi, P.C. (1989) . Energy and nutrient dynamics of above ground insects in a grassland ecosystem. Ph.D.thesis, Kumaon University, Nainital, India.

12. Kehimkar, I. 2008. The Book of Indian Butterflies.Bombay Natural History Society. OxfordUniversity Press.Mumbai.Pp 497.

13. Kunte, K. 1997. Seasonal patterns in butterfly abundance and species diversity in four tropical habitats in the northern Western Ghats.Journal of Bioscience 22: 593-60

14. Kunte, K. 2001. Butterfly diversity of Pune city along the human impact gradient. J. Ecol. Soc.,13/14: 40-45.

15. Kunte, K., Kunhikrishnan, E., Balakrishnan, M. and Susanth, C. 2008. Status and DistributionofAppiaslalage Butterfly (Lepidoptera:Pieridae) in the Western Ghats, SouthWestern India. Journal of Bombay Natural History Society, 105(3) : 354-357.

16. Larsen, T. B. 1987. The butterflies of the Nilgiri Mountains of south India. Journal of BombayNatural History Society 74: 546-549.

17. Larsen, T. B. 1988. The butterflies of the Nilgiri mountains of South India (Lepidoptera:Rhopalocera) . Journal of the Bombay Natural History Society 85: 26-43.

18. Larsen, T. B. 2002. The butterflies of Delhi, Indiaan annotated checklist. Esperiana, 9: 459479.

19. Mackinnon, P. W. and Niceville, de L. (1997) . A list of butterflies of Mussorie in the western Himalayas and neighboring region. Jour. Bombay Nat. Hist. Soc., 11: 205-211.

20. Mackinnon, P. W. and Niceville, de L. (1998) . A list of butterflies of Mussorie in the western Himalayas and neighboring region. Jour. Bombay Nat. Hist. Soc., 11: 268-289, 585-605.

21. Mudgal, V. and Hajra, P. K. (1999) .Floristic diversity and conservation strategies in India, IIII.

22. Nimbalkar, R.K., S.K. Chandekar\& S.P. Khunte (2011) . Butterfly diversity in relation to nectar food plants from BhorTahsil, Pune District, Maharashtra, India. Journal of Threatened Taxa 3(3) : 1601-1609

23. New, T. R. \& Collins, N. M. (1991). Swallowtail butterflies - an action plan for their conservation. Gland: International Union for Conservation of Nature.

24. Nair, V. P. 2005. A note on the occurrence of common Albatross, Appiasalbina (Boisduval) (Lepidoptera: Pieridae) at the St. Joseph's College Campus, Kozhikode District, Kerla. Zoos' Print Journal 20(5) : 1874.

25. Ollenbach, O. C. (1930-1931) . Butterflies collecting grounds of Mussoorie. Jour. Bombay Nat. Hist. Soc., 34:836-840.

26. Owen, D. F. (1971) .Species diversity in butterflies in a tropical garden.Biological Conservation 3(3) :191-198.

27. Padhey, A. D., Dahanukar, N., Paigankar, M.,Deshpande, M. and Deshpande, D. 2006.Season and landscape wise distribution of butterflies in Tamhini, northern Western Ghats, India. Zoos' Print J., 21: 2175-2181.

28. Pollard, E. (1990) .Monitoring butterfly numbers.Monitoring for ecology and conservation, (Ed. by F.B.Goldsmith), pp. 87-111. Chapman \& Hall, London.

29. Raut, N. B. and Pendharkar, A. 2010. Butterfly (Rhopalocera) fauna of Maharashtra Nature Park, Mumbai, Maharashtra, India. Check List 6(1) : 22-25.

30. Shields, O. (1989) .World no. of butterflies. J. Lep. Soc., 431(3) : 178-183.

31. Sidhu, AvtarKaur (2011) . Changing biodiversity scenario in the Himalayan ecosystem: Mussoorie, Uttarakhand,India, as revealed by the study of blue butterflies (Lycaenidae) . Journal of Threatened Taxa, 3(2) : 1559-1563.

32. Simpson, E. H. (1949) . Measurement of diversity. Nature, 163: 688.

33. Singh, S. (1963) .Entomological survey of Himalaya. Part XXIV - Fourth and final annotated check-list of the insects from the north-west (Punjab) Himalaya. Agra Univ. Jour. Research, 12: 363-393

34. .Singh, A.P. and Pandey, R. (2004) . A model for estimating butterfly species richness of areas across the Indian sub- continent: species proportion of Papilionidae as an Indicator. Jour Bombay Nat. Hist. Soc., Bombay,101:79-89. 
35. Singh, A. P. 2005. Initial colonization of Red Pierrot butterfly, Talicadanyseusnyseus Guerin (Lycaenidae) in the lower western Himalayas: An indicator of the changing environment. Current Science, 89 (1) , 41-42.

36. SolmanRaju, A. J. 2004. Nectar host plants of some butterfly species at Visakhapatnam. Sci.andCul., 70: 187-190.

37. Varshney R K and Smetacek P(eds.) 2015.A Synoptic Catalogue of the Butterflies of India.Butterfly Research Center,Bhimtal and IndinovPublishing,NewDelhi,ii 261pp.,8pl

38. Wynter-Blyth, M. A.(1957) . Butterflies of the Indian Region, Published by Bombay Nat. Hist. Soc., Bombay.XX+523:72 